\title{
Phase Equilibria and Phase Transformations in Molybdenum-Containing TiAl Alloys
}

\author{
Svea Mayer ${ }^{1}$, Christian Sailer ${ }^{1}$, Hirotoyo Nakashima ${ }^{2}$, Thomas Schmoelzer ${ }^{1}$, Thomas \\ Lippmann $^{3}$, Peter Staron ${ }^{4}$, Klaus-Dieter Liss ${ }^{5}$, Helmut Clemens ${ }^{1}$ and Masao Takeyama ${ }^{2}$ \\ ${ }^{1}$ Department of Physical Metallurgy and Materials Testing, Montanuniversität Leoben, Leoben, \\ Austria \\ ${ }^{2}$ Department of Metallurgy and Ceramics Science, Tokyo Institute of Technology, Tokyo, Japan \\ ${ }^{3}$ Institute of Materials Research, Helmholtz-Zentrum Geesthacht Outstation at DESY, Hamburg, \\ Germany \\ ${ }^{4}$ Institute of Materials Research, Helmholtz-Zentrum Geesthacht, Geesthacht, Germany \\ ${ }^{5}$ The Bragg Institute, Australian Nuclear Science and Technology Organisation, Lucas Heights, \\ Australia
}

\begin{abstract}
Molybdenum, being a strong $\beta$ stabilizer, is an important alloying element in TiAl alloys, since a significant volume fraction of the disordered bcc $\beta$-phase at elevated temperatures improves the processing characteristics during hot-working. Unfortunately, the effect of Mo on the individual phases and their transition temperatures is not completely known but is necessary for designing engineering applications. In this paper, sections of the Ti-Al-Mo ternary phase diagram derived from thermodynamic calculations as well as experimental data are presented. Further, the phase transition temperatures given by the phase diagrams are compared with results from isothermal heat treatment studies, differential scanning calorimetry measurements and insitu high-temperature diffraction experiments. Combining all of these results, a revised phase diagram is proposed.
\end{abstract}

\section{INTRODUCTION}

Due to their attractive properties, intermetallic TiAl alloys are considered for hightemperature applications in aerospace and automotive industries. Their advantages are mainly seen in low density (3.9- $4.2 \mathrm{~g} / \mathrm{cm}^{3}$, depending on composition and constitution), high specific yield strength, high specific stiffness, good oxidation resistance, resistance against "titanium fire", and good creep properties up to high temperatures [1]. One recent approach is the design of so-called $\beta / \gamma$-alloys with microstructures in which homogeneously distributed $\beta$ - and $\gamma$-phases are the main constituents [2]. Based on the fact that Mo is a strong $\beta$ stabilizer, the ternary system Ti-Al-Mo is well-suited for studying this type of alloys. In this paper, which is based on a recent study [3], representative sections of the ternary Ti-Al-Mo phase diagram, derived from thermodynamic calculations and experimental results, are presented. In principle, advanced multi-phase TiAl alloys contain three intermetallic phases, namely $\gamma, \alpha_{2}$ and $\beta_{0}$, which are all ordered at ambient temperature. An increase of temperature induces that the ordered hexagonal $\alpha_{2}$-phase transforms to the disordered hexagonal $\alpha$ and the ordered $\beta_{\mathrm{o}}$-phase transforms to the disordered bec $\beta$-variant. For the prediction of the constituent phases and related transition temperatures, thermodynamic calculations based upon the CALPHAD method were conducted. For this purpose, the software package MatCalc was employed using a commercial TiAl database [4]. In order to critically evaluate the thermodynamically calculated as well as the experimental phase diagram taken from literature, two model alloys with a nominal composition 
of Ti-44Al-3Mo and Ti-44Al-7Mo (in at $\%$ ) were investigated, on which isothermal short- and long-term heat treatments were employed, followed by water quenching. All these heat-treated samples were subsequently analyzed by means of scanning electron microscopy (SEM). The determination of the phase evolution and phase transition temperatures using in-situ high-energy X-ray diffraction (HEXRD) experiments [5] were conducted during continuous heating, whereas the verification of the obtained phase transition temperatures was performed via additional differential scanning calorimetry (DSC) investigations. Finally, the experimentally obtained and calculated results are compared to the quasi-binary phase diagrams, differences are discussed and a revised phase diagram is proposed.

\section{EXPERIMENTAL DETAILS}

The present investigations were carried out on two Mo-containing TiAl alloys, Ti-44Al3Mo and Ti-44Al-7Mo, provided by GfE Metalle und Materialien GmbH, Nuremberg, Germany, in the form of hot-isostatically pressed (HIP) cylindrical castings with a length of $200 \mathrm{~mm}$ and a diameter of $53 \mathrm{~mm}$. The HIP process, accompanied by a slow cooling rate to room temperature, was performed at $1200^{\circ} \mathrm{C}$ for $240 \mathrm{~min}$ at a maximum pressure of $200 \mathrm{MPa}$, in order to eliminate casting defects such as gas porosity to provide near-equilibrium conditions. The actual chemical composition as obtained by X-ray fluorescence spectroscopy is Ti-44.58 Al-3.23 Mo-0.12 B and Ti-43.94 Al-6.78 Mo-0.09 B (in at\%). The small amount of B is intended to refine the microstructure due to the formation of borides during solidification. Microstructural investigations were performed by SEM in order to determine the morphology and constitution of the appearing phases. All images were acquired with a Zeiss EVO50, Germany, which is equipped with an Inca Dry Cool energy-dispersive X-ray spectrometer (EDS) from Oxford Instruments, UK, for analytical measurements. The SEM investigations were carried out in backscattered electron (BSE) mode at an acceleration voltage of $15 \mathrm{kV}$ on the specimens prepared by standard metallographic methods. A quantitative evaluation of the phases present as a function of temperature using HEXRD [6] was performed at the HARWI II beamline at the storage ring DORIS III at DESY in Hamburg, Germany, which is operated by the Helmholtz-Zentrum Geesthacht [7], where the beam defining an area of $0.5 \times 0.5 \mathrm{~mm}^{2}$ had a mean energy of 104.7 $\mathrm{keV}$. Solid samples with a diameter of $5 \mathrm{~mm}$ and a length of $15 \mathrm{~mm}$ were rapidly inductionheated to $1000^{\circ} \mathrm{C}$ in a custom built furnace under Ar atmosphere, held at this temperature for 2 min and then continuously heated to $1350^{\circ} \mathrm{C}$ with a heating rate of $2{ }^{\circ} \mathrm{C} / \mathrm{min}$. The temperature was controlled using a differential pyrometer. A mar555 area detector from MAR Research, Norderstedt, Germany, was used for recording several complete diffraction rings. Studying the sequence of phase transformations, DSC analyses were performed with a Setaram LabSys evo system on samples with a mass of about $30 \mathrm{mg}$. The experiments were carried out from room temperature to $1500^{\circ} \mathrm{C}$ at different heating rates using covered $\mathrm{Al}_{2} \mathrm{O}_{3}$ crucibles with sapphire inserts. The occurring phase transition temperatures were evaluated by extrapolating to a heating rate of zero. The thereby obtained $\gamma$ dissolution temperature $\left(\mathrm{T}_{\gamma}\right)$ was used for correcting the temperature measurement of the in-situ HEXRD experiments since the pyrometer is extremely error-sensitive to surface-related processes such as oxide-scale formation at high temperatures. For the prediction of the prevailing phases and their transition temperatures thermodynamic equilibrium calculations based on CALPHAD (calculation of phase diagrams) were conducted with the software MatCalc using a commercially available TiAl database [4]. 


\section{RESULTS AND DISCUSSION}

A thermodynamically calculated section of the ternary phase diagram Ti-Al-Mo for a constant $\mathrm{Al}$ content of 44 at $\%$ and Mo contents in the range of $0-10$ at $\%$ is displayed in Figure 1a, where the two vertical lines indicate the Mo contents of the alloys investigated. As the diagram shows, both alloys solidify entirely via the bcc $\beta$-phase. Thus, the solidification pathway from the liquid to the stable solid for alloy Ti-44Al-3Mo is supposed to be: $\mathrm{L} \rightarrow \mathrm{L}+\beta \rightarrow \beta \rightarrow$ $\alpha+\beta \rightarrow \alpha+\beta+\gamma \rightarrow \alpha_{2}+\beta+\gamma$. The transition $\alpha+\beta+\gamma \rightarrow \alpha_{2}+\beta+\gamma$ occurs at the eutectoid temperature. According to Figure 1a alloy Ti-44Al-7Mo solidifies as follows: $\mathrm{L} \rightarrow \mathrm{L}+\beta \rightarrow \beta \rightarrow \beta+\gamma \rightarrow$ $\alpha_{2}+\beta+\gamma$. At this point, it should be mentioned that the thermodynamic simulations indicate no disorder/order temperature for the $\beta \leftrightarrow \beta_{\mathrm{o}}$ reaction.
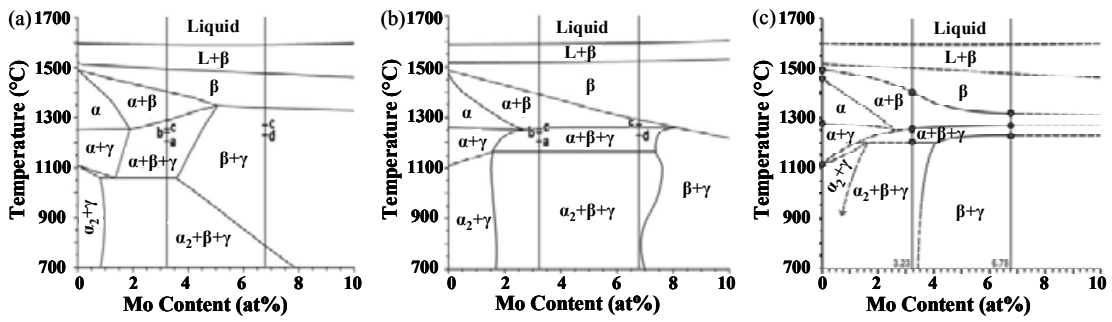

Figure 1. (a) Calculated section of the ternary phase diagram Ti-44Al-(0-10)Mo [3]; (b) Section of the ternary Ti-44Al-(0-10)Mo phase diagram established from literature data [8-11]. In (a) and (b) the investigated alloys Ti-44Al-3Mo and Ti-44Al-7Mo are marked by vertical lines. The transition temperatures as determined by in-situ HEXRD diffraction are indicated by horizontal marks and corresponding letters: "a" indicates the $\alpha_{2} \rightarrow \alpha$ disordering temperature, "b" the $\beta_{0} \rightarrow$ $\beta$ disordering temperature and "c" the $\gamma$ dissolution temperature. The letter " $\mathrm{d}$ " stands for the onset of the $\alpha$-phase formation upon heating of Ti-44Al-7Mo. (c) Revised phase diagram as an outcome of this study which also includes the results of isothermal short- and long-term heat treatments as well as DSC measurements.

Figure $1 \mathrm{~b}$ displays a section of the Ti-Al-Mo ternary phase diagram obtained experimentally for the same Al concentrations as published in [8-11]. As in the case of Figure 1a, no distinction between the ordered and disordered $\beta$-phases was made. The solidification sequence of alloy Ti-44Al-3Mo is equal to the one found in the thermodynamic simulation, but with the difference that both alloys show the same solidification and transformation pathway, which is in contrast to Figure 1a. Accordingly, there are also other substantial differences between the two phase diagrams. For a Mo concentration $<1.5$ at $\%$, for instance, the calculation predicts that Mo stabilizes $\alpha$ against $\alpha_{2}$ (Figure 1a), whereas Figure 1b shows the opposite behavior, i.e. Mo stabilizes the ordered $\alpha_{2}$-phase against the disordered one. Such a behavior was also found for the system Ti-Al-V [12]. Another pronounced deviation exists where the $\alpha \leftrightarrow \alpha_{2}$ reaction occurs. The calculated temperature is about $1060^{\circ} \mathrm{C}$, whereas the experimental data from literature indicate an increase of this reaction to $1165^{\circ} \mathrm{C}$. However, it should be mentioned that previous thermodynamic calculations of transition temperatures for other highly $\beta$-stabilized $\mathrm{TiAl}$ alloys also showed a strong deviation from experimental data, especially with respect to the 
eutectoid temperature [13]. Moreover, in the experimental phase diagram (Figure 1b), the lower boundary of the single $\beta$-phase field region, particularly with regards to the transformation point in which the $\beta$-phase meets with the three other phase-field regions, is shifted on the one hand to a lower temperature and on the other hand to a significantly higher Mo concentration. Therefore, in the case of Ti-44Al-7Mo the $\alpha / \alpha_{2}$-phase is present below $1295^{\circ} \mathrm{C}$ according to Figure $1 \mathrm{~b}$, whereas in the thermodynamic calculation shown in Figure 1a the $\alpha_{2}$-phase is predicted to be stable only below $790^{\circ} \mathrm{C}$. This in turn means that for this alloy composition the absence of the $\alpha / \alpha_{2}$-phase in the thermodynamic simulation at elevated temperatures precludes the occurrence of the eutectoid reaction. Furthermore, a discrepancy in the prediction for $\mathrm{T}_{\gamma}$ of the two phase diagrams was observed, supporting the decision to revise the Ti-Al-Mo phase diagram by incorporating the results of this study (see Figure 1c).

The individual microstructural features of the two investigated alloys in the as-HIP condition and after annealing at $1000^{\circ} \mathrm{C}$ for 30 days with subsequent water quenching were characterized by SEM. Figure 2a displays the SEM image of the HIP Ti-44Al-3Mo. It can be seen that the microstructure consists of globular $\gamma$-grains and lamellar $\left(\gamma+\alpha_{2}\right)$-colonies in which a small volume fraction of $\beta_{\mathrm{o}}$ is embedded. However, the majority of $\beta_{\mathrm{o}}$ is still located along the grain boundaries. EDS analyses give information that the $\beta_{0}$-phase is enriched in Mo when compared to the other prevailing phases. Figure $2 \mathrm{~b}$ shows the HIP alloy Ti-44Al-7Mo, where former $\beta$-grains are intersected by elongated $\gamma$-grains, which were formed during a solid state phase transformation. Moreover, a small volume fraction of equiaxed $\alpha_{2}$-grains is visible. As expected, the $\beta_{0}$-phase fraction at room temperature of Ti-44Al-7Mo is significantly higher when compared to the alloy Ti-44Al-3Mo.
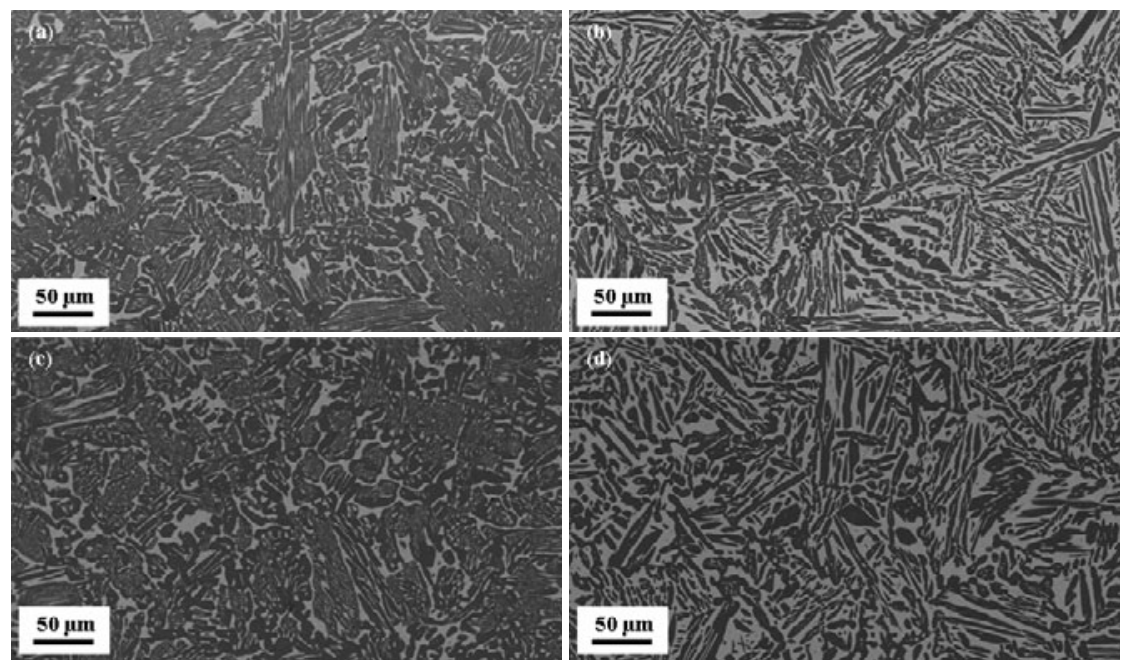

Figure 2. SEM images of Ti-44Al-3Mo (left) and Ti-44Al-7Mo (right) taken in BSE mode, wherein the $\beta_{0}$-phase shows the brightest contrast, the $\gamma$-phase appears dark and the $\alpha_{2}$-phase exhibits a light-gray contrast. (a) and (b): as-HIP condition; (c) and (d): after annealing at $1000^{\circ} \mathrm{C}$ for 30 days. 
Images of the samples annealed at $1000^{\circ} \mathrm{C}$ for 30 days are displayed in Figures $2 \mathrm{c}$ and $\mathrm{d}$. Obviously, the long-term annealing has led to a coarsening of the lamellar colonies in Ti-44Al$3 \mathrm{Mo}$ (Figure 2c), but no other significant differences to the as-HIP condition can be observed. However, SEM and XRD analyses have clearly shown that the annealed Ti-44Al-3Mo sample consists of $\gamma, \alpha_{2}$, and $\beta_{0}$-phase whereas only $\gamma$ and $\beta_{0}$-phase are present in the long-term annealed Ti-44Al-7Mo (Figure $2 d$ ). From this result it is evident, that the extension of the $\left(\alpha_{2}+\beta+\gamma\right)$-phase field region in Figure $1 \mathrm{~b}$ is not represented correctly. Concerning the evolution of the microstructure during solidification, HIP as well as short- and long-term ageing treatments further investigations are underway.

The phase evolution as a function of temperature resulting from in-situ HEXRD experiments conducted on Ti-44Al-Mo is exemplarily shown for a Mo content of 7 at $\%$ in Figure 3 . According to this diagram only a negligible fraction of $\alpha / \alpha_{2}$-phase exists up to temperatures of about $1200^{\circ} \mathrm{C}$, which is in accordance with the phase diagrams shown in Figures 1a and b. But this finding is in contradiction to the result of the long-term annealing at $1000^{\circ} \mathrm{C}$ where no $\alpha_{2}$ was found (Figure 2d). However, it must be noted at this point, that after the HIP process the material is not in thermodynamic equilibrium. Thus, at low temperatures, where diffusion processes are very slow over long distances, the phase fractions determined by continuous heating experiments at a rate of $2{ }^{\circ} \mathrm{C} / \mathrm{min}$ may not correspond to the equilibrium conditions that are reflected in the phase diagram. Therefore, especially at low temperatures, phases might be observed that are thermodynamically unstable and only present because of their slow kinetics of phase transformation or dissolution processes. As a matter of fact, Figure 3 shows that the volume fraction of the $\alpha / \alpha_{2}$-phase decreases with increasing temperature to almost zero $\left(\sim 1200^{\circ} \mathrm{C}\right)$ until at around $1230^{\circ} \mathrm{C}$ a distinct increase in the $\alpha / \alpha_{2}$-phase fraction is observed. As mentioned above, the dissolution of $\alpha / \alpha_{2}$-phase below $\sim 1230^{\circ} \mathrm{C}$ is in agreement with the result of the long-term annealing treatment. Thus, the $\left(\alpha_{2}+\beta+\gamma\right)$-phase field region below the $\alpha_{2} \rightarrow \alpha$ temperature as shown in Figure $1 \mathrm{~b}$ cannot be correct.

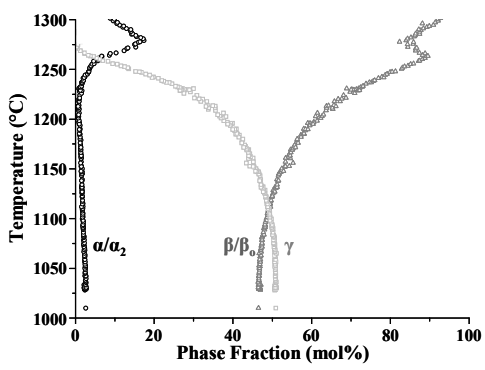

Figure 3. Phase evolution as a function of temperature resulting from in-situ HEXRD experiments of Ti-44Al-7Mo [3]. Circles stands for the $\alpha$-phase, whereas triangles and squares represent the $\beta$ - and $\gamma$-phase, respectively.

Further investigations were performed to determine the phase transition temperatures of alloy Ti-44Al-3Mo [3]. By means of HEXRD the $\alpha_{2} \rightarrow \alpha$ temperature was determined to be at $\sim 1205^{\circ} \mathrm{C}$, which is significantly higher than that predicted by the calculated phase diagram $\left(\sim 1058^{\circ} \mathrm{C}\right)$ in Figure 1a. In consideration of Figure $1 \mathrm{~b}\left(\sim 1160^{\circ} \mathrm{C}\right)$ the difference in temperature was smaller, but still noticeable. Taking into account all the experimental data from this study, 
i.e. obtained from heat treatments, DSC and HEXRD, a revised phase diagram for Ti-44Al-Mo is proposed as shown in Figure 1c. According to this diagram, the following solidification pathway is expected for Ti-44Al-3Mo: $\mathrm{L} \rightarrow \mathrm{L}+\beta \rightarrow \beta \rightarrow \alpha+\beta \rightarrow \alpha+\beta+\gamma \rightarrow \alpha_{2}+\beta+\gamma$, while alloy Ti-44Al7 Mo solidifies and transforms according to $L \rightarrow L+\beta \rightarrow \beta \rightarrow \alpha+\beta \rightarrow \alpha+\beta+\gamma \rightarrow \beta+\gamma$. In-situ HEXRD was also used to determine the disordering temperature of the $\beta_{\mathrm{o}}$-phase during continuous heating [3]. According to these investigations disordering takes place for Ti-44Al$3 \mathrm{Mo}$ at approximately $1240^{\circ} \mathrm{C}$.

\section{SUMMARY}

Sections of the Ti-44Al-(0-10)Mo ternary phase diagram were established from thermodynamic equilibrium calculations as well as from experimental data published in literature. The phase diagrams obtained in this way exhibit significant discrepancies concerning the extension of the phase field regions as well as the phase transition temperatures. In order to propose a revised phase diagram, long-term heat treatments, DSC measurements and in-situ diffraction experiments with synchrotron radiation were carried out on two alloys with a nominal composition of Ti-44Al-3Mo and Ti-44Al-7Mo.

\section{ACKNOWLEDGMENTS}

A part of this study was conducted within the framework of the BMBF project O3X3530A, Germany, and the Styrian Materials Cluster, Austria.

\section{REFERENCES}

1. H. Kestler and H. Clemens in Titanium and Titanium Alloys, edited by C. Leyens and M. Peters (Wiley-VCH, Weinheim, Germany, 2003) p. 351.

2. T. Schmoelzer, S. Mayer, F. Haupt, G. A. Zickler, C. Sailer, L. Lottermoser, V. Güther, K.-D. Liss, and H. Clemens, Mater. Sci. Forum 654-656, 456 (2010).

3. T. Schmoelzer, S. Mayer, C. Sailer, F. Haupt, V. Güther, P. Staron, K.-D. Liss, and H. Clemens, Adv. Eng. Mater., (2010) (accepted for publication).

4. N. Saunders in Gamma Titanium Aluminides, edited by Y.-W. Kim, D. M. Dimiduk and M. H. Loretto (TMS, Warrendale, PA, 1999) p. 183.

5. K.-D. Liss, A. Bartels, A. Schreyer, and H. Clemens, Texture. Microstruct. 35, 219 (2003).

6. L. A. Yeoh, K.-D. Liss, A. Bartels, H. F. Chladil, M. Avdeev, H. Clemens, R. Gerling, and T. Buslaps, Scripta Mater. 57, 1145 (2007).

7. P. Staron, N. Schell, A. Haibel, F. Beckmann, T. Lippmann, L. Lottermoser, J. Herzen, T. Fischer, M. Koçak, and A. Schreyer, Mater. Sci. Forum 638-642, 2470 (2010).

8. T. Hamajima, G. Luetjering and S. Weissmann in Red Books XVIII (Kombinat Viniti, Moscow, Russia, 1972).

9. R. Hansen and R. Aravamudham, Z. Metallkd. 61, 115 (1970).

10. A. K. Singh and D. Banerjee, Metall. Mater. Trans. A 28, 1745 (1997).

11. G. Czi-Min and E. Pylaeva in Red Books IX (Kombinat Viniti, Moscow, Russia, 1963).

12. M. Takeyama and S. Kobayashi, Intermetallics 13, 993 (2005).

13. H. F. Chladil, H. Clemens, G. A. Zickler et al., Int. J. Mater. Res. 98, 1131 (2007). 